\title{
Fallopian Tube Cancer by AJCC v6 Stage
}

National Cancer Institute

\section{Source}

National Cancer Institute. Fallopian Tube Cancer by AJCC v6 Stage. NCI Thesaurus. Code C90499.

A term that refers to the staging of fallopian tube carcinoma according to the American Joint Committee on Cancer, 6th edition. 